\title{
ECG Signal Classification Review
}

\author{
Muhammad Rausan Fikri ${ }^{1}$, Indah Soesanti ${ }^{2}$, Hanung Adi Nugroho ${ }^{3}$
}

\begin{abstract}
The heart is an important part of the human body, functioning to pump blood through the circulatory system. Heartbeats generate a signal called an ECG signal. ECG signals or electrocardiogram signals are basic raw signals to identify and classify heart function based on heart rate. Its main task is to analyze each signal in the heart, whether normal or abnormal. This paper discusses some of the classification methods which most frequently used to classify ECG signals. These methods include pre-processing, feature extraction, and classification methods such as MLP, K-NN, SVM, CNN, and RNN. There were two stages of ECG classification, the feature extraction stage and the classification stage. Before ECG features were extracted, raw ECG signal data first processed in the pre-processing stage because ECG signals were not necessarily free of noise. Noise will cause a decrease in accuracy during the classification process. After features were extracted, ECG signals were then classified with the classification method. Neural Network methods such as CNN and RNN are best to use since they can give better accuracy. For further research, the machine learning method needs to be improved to get high accuracy and high precision in the ECG signals classification.
\end{abstract}

Keyword-Electrocardiogram, Neural Network, Deep Learning, Classification Algorithm, Signal Processing.

\section{INTRODUCTION}

The heart is one of the most important parts of the human body; it pumps blood to all parts of the human body through the circulatory system [1]. Therefore, the heart also functions as a supplier of oxygen and nutrients to other body tissues. Blood pumped by the heart also carries carbon dioxide and other useless substances to be discharged out of the body; if the heart has a problem and cannot function properly, it will have a fatal impact on the body.

Heart disease (coronary) is one of the biggest causes of death globally, including in Indonesia. According to data from the IHME (Institute for Health Metrics and Evaluation), most deaths are caused by heart and blood vessel-related diseases such as Cardiovascular. Data in 2016 showed that more than 17 million people or around $32.26 \%$ of all deaths in the world were caused by heart disease, $63 \%$ of deaths due to cardiovascular disease were sufferers by 70 years old, $29.13 \%$ by 50 - 69 years old, and $7.61 \%$ by the age of $15-49$ years old. Based on these data, heart disease is the biggest cause of death more than cancer and other internal diseases [2]. Therefore, the heart is a vital organ in which health needs to be maintained.

A tool used to read ECG signals is electrocardiography. It functions to determine the condition of the heart. When the

1,2,3 Department of Electrical and Information Engineering, Faculty of Engineering, Universitas Gadjah Mada, Jln. Grafika No. 2, Kampus UGM, Yogyakarta, 55281, INDONESIA (Tel. +62274552305; e-mail: $\quad{ }^{1}$ rausanfikri@mail.ugm.ac.id, 2indahsoesanti@ugm.ac.id, ${ }^{3}$ adinugroho@ugm.ac.id) heart pumps blood throughout the body, the heart produces an electrical signal called an electrocardiogram (ECG). The signal is then captured using electrocardiography, which doctors will use to diagnose illnesses suffered by patients. ECG signals have a consistent pattern for a healthy heart (normal), while inconsistent signals can be a sign of heart problems (abnormalities) [2]. Based on the ECG signal form in Fig. 1, various kinds of abnormalities in the heart can be diagnosed. All kinds of variations in ECG signal waves, such as an increase in amplitude or decrease in amplitude and the wave inconsistency, allow the doctor to diagnose abnormalities in the heart.

The electrocardiogram is a test to measure electrical activity in the heart. Raw signal from ECG is used to identify and classify heart signal based on heart rate. Its main task is to analyze whether the heart's signals are normal or abnormal on every ECG signal recorded [3]. An abnormal form of a regular signal, amplitude, or irregular time interval will result in arrhythmias. In order to detect arrhythmias in ECG signals automatically, the steps are divided into two, namely feature extraction and signal classification. However, ECG signals also cannot be ascertained free of noise. Therefore, before extracting features, the raw signal first enters the pre-processing stage. Several filtering procedures filter the raw ECG signal during the pre-processing stage to eliminate noise interference. After the filtered signal is free from noise, the feature will be extracted from the signal, and then the features will be selected. Based on selected features, the signal will be classified using the ECG signal classification method, which will help to diagnose patients accurately.

Not all the ECG extracted features are useful; some features may not provide the appropriate information for the correct signal classification. Therefore, it is highly recommended to identify and select the best features that will help with classification; selected best features will help to reduce the computational and complexity analysis. There are many feature extraction methods, and feature selection methods developed to ensure the classification method accuracy.

The same ECG signal can be interpreted differently by different doctors. Therefore, an intelligent system that can conduct electrocardiogram data analysis and classify the signal patterns accurately is used. It is done so that fatal errors due to incorrect interpretation of ECG signal readings can be avoided. Heart signals are detected using a biopotential sensor. It functions as a transducer of the body's ionic current into a conducting electron current, processed by an electrocardiograph. A computer then processes the ECG signal from the electrocardiograph processing to get a ready-toprocess signal. After that, the signals will be trained on artificial neural networks. Subsequently, they will be able to identify and classify the heart signals that can provide the results of a 


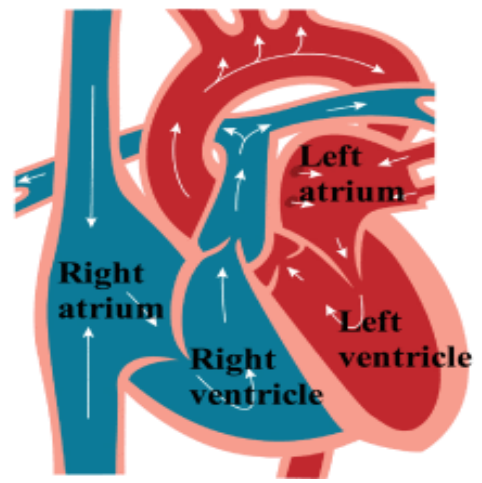

(a)

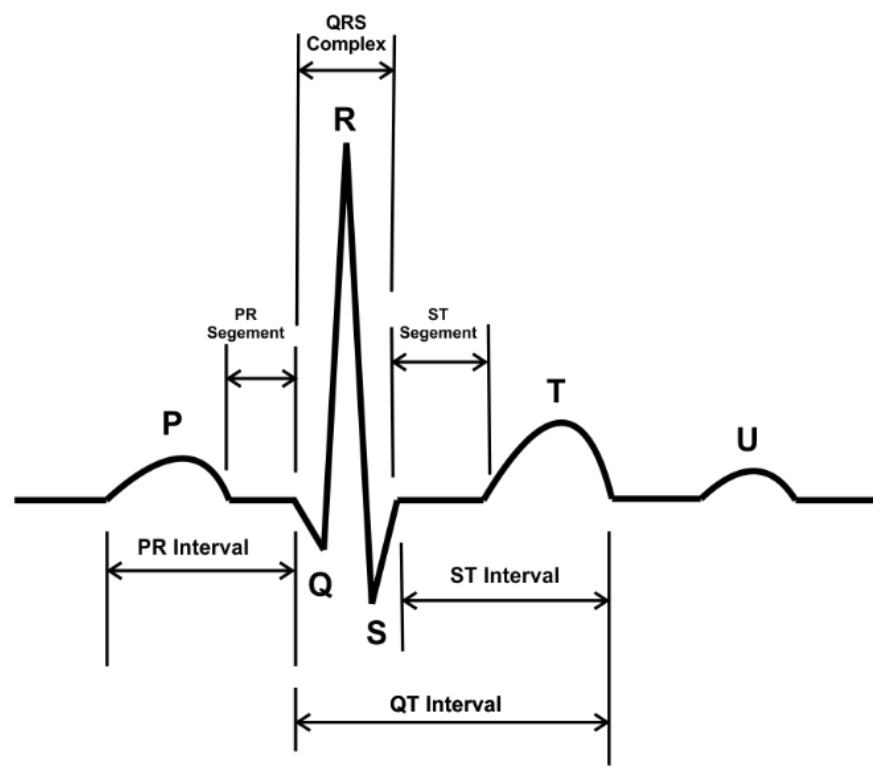

(b)

Fig. 1(a) Heart illustration, (b) ECG signal.

differential diagnosis for doctors. This study aims to analyze signals using machine learning methods to improve classification accuracy and precision for multiple classes based on this background.

\section{LITERATURE REVIEW}

Many kinds of research had been previously conducted to analyze and classify ECG signals. Previous researchers used various kinds of analysis techniques and algorithms to precisely identify ECG signals. Researchers' studies on [4], [5] introduce a power spectrum analysis method for ECG signals. Other techniques such as PCA (principal component analysis) to measure ECG signals conducted in the study [6], Hilbert transformation analysis [7], and adaptive filtering [8] were widely used in other studies. Other studies also used Markov models [9] and the neural network's method [10]. Reference [11] examined the recognition of the normal type of ECG pattern with the percentage accuracy of recognizing ECG patterns reaching $92.5 \%$. In [12] the classification and analysis of ECG signals using wavelet transforms and backpropagation neural networks have been examined.
Research on ECG signal classification using neural network methods has proven to be able to get high accuracy, especially for solving non-linear problems. Artificial Neural Network or ANN has been proven as an efficient method [13]; hence, many ANN approaches were proposed for ECG signal and wavelet analysis as in the study [14]-[16]. Research to recognize the frequency characteristics of arrhythmias using artificial neural networks was carried out, and it obtained relatively high results [17]. However, these studies only focus on recognizing frequency characteristics and have not used large numbers of datasets. Research on ECG signals through the frequency domain has also been carried out using the Compressive Neural Network method [18]. Research using frequency domains is different from using time-domain analysis as frequency domain analysis requires fewer parameters, less pre-processing, and less analysis complexity. The experimental results show that compared to conventional ECG wavelet analysis, the proposed method can save up to $80 \%$ of ECG diagnosis time [18]. However, this research still has not optimized the selection of features to improve the classification accuracy.

Proposed solutions based on neural networks are very popular to use, especially in recent years. Deep neural network methods such as CNN and RNN are mostly used to improve classification accuracy. These methods automatically extract their own features from the dataset so that they can obtain higher accuracy than conventional methods. It is because the feature extraction method from the deep neural network is more robust against variations of various ECG waveforms [19]. Therefore, based on research that has been done before, methods for the classification of ECG signals using a neural network have been widely proposed. Deep neural network methods such as convolutional neural networks and recurrent neural networks have become popular and often used because these methods have proven to be efficient for handling ECG signal classification.

\section{ECG CLASSIFICATION METHOD}

The methods used to classify ECG signals are very diverse. However, today, the frequently used methods are Support Vector Machine (SVM), Multi-Layer Perceptron (MLP), KNN, Convolutional Neural Network (CNN), and Recurrent Neural Network (RNN) methods. Deep neural network methods such as CNN and RNN are more popular. Frequently used public ECG datasets are available at the Physionet database.

\section{A. ECG Signal Processing Stage}

Generally, ECG signal processing stages are data acquisition, pre-processing, feature extraction and feature selection, and finally, classification, as seen in Fig. 2.

1) Pre-processing Stage: The pre-processing stage aims to free the ECG signal from noise and prepare the raw data for processing. Usually, firstly, a raw ECG signal will be cleaned from noise and then segmented, as seen in Fig. 3. After the data goes through the pre-processing process and free of noise, it will enter the feature extraction stage. 


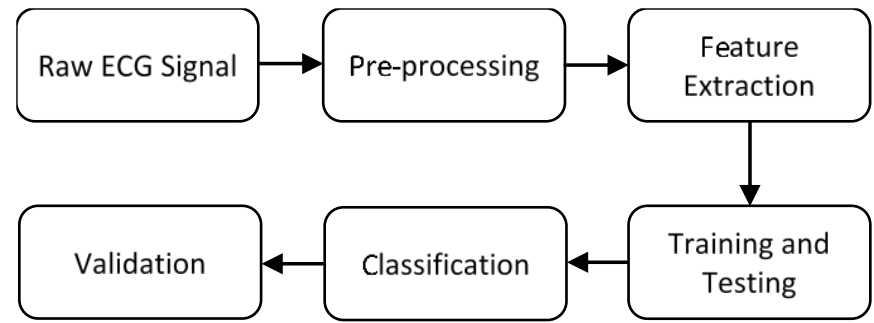

Fig. 2 ECG signal processing stage.

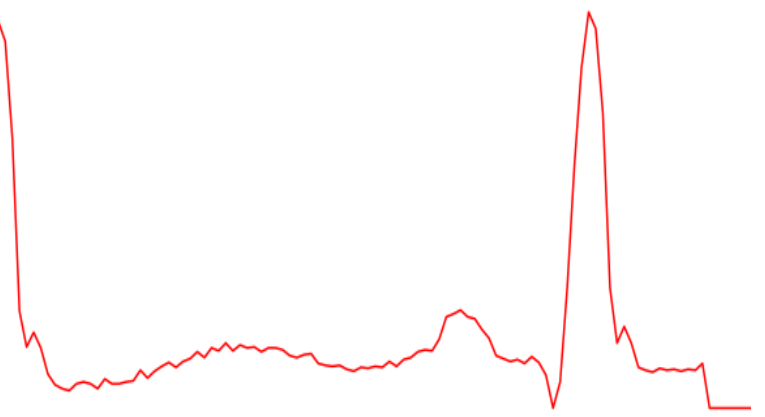

Fig. 3 Segmented ECG signal.

2) Feature Extraction Stage: Feature extraction of ECG signals is obtained by decomposing the ECG signal into several parts, like extracting Q-, R-, T-, and U- waves in the signal. Wavelet transform is a frequently used feature extraction method, but deep learning methods such as CNN can automatically extract the feature.

3) Training and Testing Stage: At the stage of training and testing, the important features from the ECG signal are selected. The best or most influential feature on the classification will affect the accuracy. Machine learning method such as SVM, KNN, or MLP is often used, but neural network method such as CNN and RNN can give higher accuracy.

4) Classification Stage: In this stage, ECG signals will then be grouped based on the results obtained by the classification method.

5) Validation Stage: The last stage is validation. It will determine whether the proposed model has achieved adequate accuracy with the training dataset that has been given. A confusion matrix is one of the frequently used validation techniques.

\section{B. Artificial Neural Network}

An Artificial Neural Network (ANN) or Neural Network is a technique or method for processing information with certain performance characteristics similar to biological neural networks. The backpropagation neural network includes three stages, namely forward propagation, backpropagation, and weight change. There are two main learning parameters in reverse propagation, namely the learning rate $\alpha$ and momentum $\mu$. Learning rate is used to adjust the learning speed and avoid predominant weight changes due to different data from the others; momentum is used [20]. The architecture of an ANN is shown in Fig. 4.

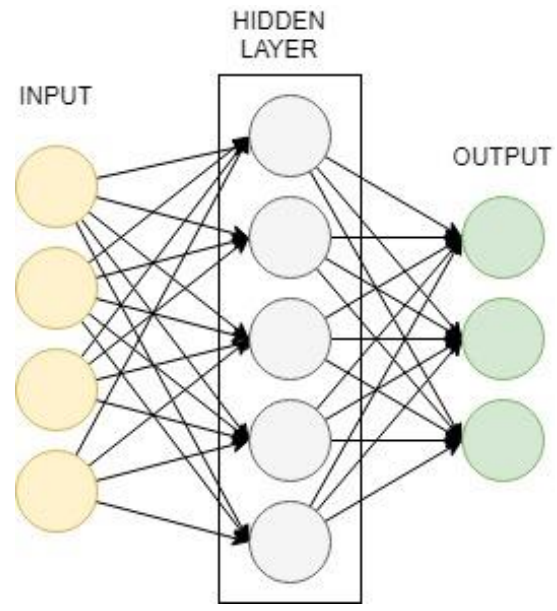

Fig. 4 ANN architecture.

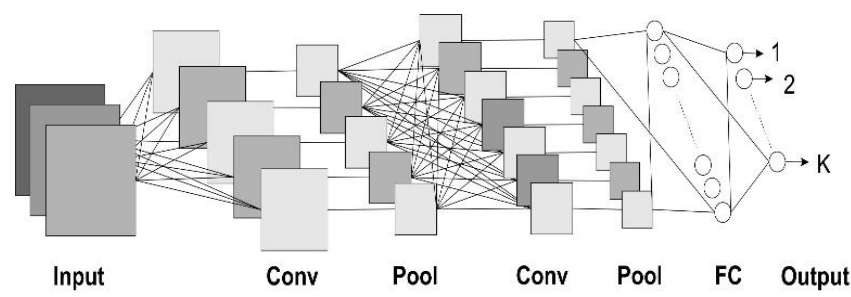

Fig. $5 \mathrm{CNN}$ architecture.

For ECG signal classification, ANN is often used as a classification method as in [14], [15]. First, they use the wavelet transform method to decompose the signal. After that, the signal will be feed to a neural network for classification.

\section{Convolutional Neural Network}

$\mathrm{CNN}$ is a feedforward network, which means the flow of information occurs in one direction only, from input to output. Like ANN, which is inspired by the brain's neural network, the brain's visual cortex consists of layers - complex and simple. CNN architecture also has layers that generally consist of Convolutional layers and pooling layers [10].

Data on CNN usually in the form of gray-scale images (one channel) or RGB images (three channels). The architecture on CNN can be seen in Fig. 5. Several convolutional layers or pooling layers with or without activation functions in $\mathrm{CNN}$ follow the input layer. For CNN classification, one or more full connection layers (FC) can be used. The last layer produces predictive values [21].

Every layer of $\mathrm{CNN}$ has a certain activation function that controls the number of output values that will spread to the next layer. In middle layer have a rectified linear unit (ReLU) often used as

$$
f\left(a_{i}^{l}\right)=\max \left(0, a_{i}^{l}\right) .
$$

Note that $a_{i}^{l} \in \mathrm{R}, R$ is the number of signals received by the $i$ unit in the middle-l layer. Meanwhile, for the last layer, softmax function.

$$
f_{k}(z)=\frac{\exp \left(z_{k}\right)}{\sum_{k=1}^{K} \exp \left(z_{k}\right)} .
$$

Equation (2) is often used to get possibilities from the output. 
TABLE I

RESEARCH COMPARISON OF ECG CLASSIFICATION

\begin{tabular}{|c|c|c|c|c|}
\hline Paper & Dataset & Pre-Processing & $\begin{array}{l}\text { Feature Extraction and } \\
\text { Classification Method }\end{array}$ & Result \\
\hline$[22]$ & MIT-BIH Database & - & $\begin{array}{l}\text { Feature extraction and classifier } \\
\text { using CNN method }\end{array}$ & $\begin{array}{l}\text { The proposed method gets } 93.6 \% \\
\text { accuracy }\end{array}$ \\
\hline$[23]$ & $\begin{array}{l}\text { PhysioBank.com, } \\
\text { Kaggle.com }\end{array}$ & - & $\begin{array}{l}\text { Feature extraction and classifier } \\
\text { using CNN method }\end{array}$ & $\begin{array}{l}\text { The proposed method gets } 83.5 \% \\
\text { accuracy. }\end{array}$ \\
\hline$[24]$ & INCART database & $\begin{array}{l}\text { By utilizing SMOTE } \\
\text { algorithm to synthesize } \\
\text { heartbeats for the class } \\
\text { with fewer instance }\end{array}$ & TD-CNN & $\begin{array}{l}\text { The proposed method gets } 99.1 \% \\
\text { accuracy, } 99.3 \% \text { sensitivity, and } \\
98.5 \% \text { specificity. }\end{array}$ \\
\hline$[26]$ & $\begin{array}{l}\text { MIT-BIH ECG } \\
\text { arrhythmia database }\end{array}$ & $\begin{array}{l}\text { Segmentation of heartbeat } \\
\text { using Pan-Tompkin's } \\
\text { algorithm }\end{array}$ & $\begin{array}{l}\text { Wavelet transforms for feature } \\
\text { extraction. RNN for } \\
\text { classification }\end{array}$ & $\begin{array}{l}\text { The proposed method obtains } \\
99 \% \text { accuracy, } 95 \% \text { sensitivity, } \\
\text { and } 99.8 \% \text { specificity. }\end{array}$ \\
\hline$[27]$ & $\begin{array}{l}\text { MIT-BIH } \\
\text { arrhythmia database }\end{array}$ & Segmentation & $\begin{array}{l}\text { Feature Extraction using spike- } \\
\text { timing-dependent plasticity } \\
\text { (STDP). Classification using } \\
\text { reward-modulated STDP } \\
\text { method (R-STDP) }\end{array}$ & $\begin{array}{l}\text { The proposed method obtains } \\
97 \% \text { accuracy }\end{array}$ \\
\hline [28] & $\begin{array}{l}\text { PAF prediction challenge } \\
\text { database }\end{array}$ & $\begin{array}{l}\text { The signal is segmented } \\
\text { into six }\end{array}$ & $\begin{array}{l}\text { Feature extraction using CNN } \\
\text { and then integrated with other } \\
\text { standard classifiers, K-NN, } \\
\text { SVM, and the multilayered } \\
\text { perceptron (MLP) }\end{array}$ & $\begin{array}{l}\text { K-NN classifier, which integrated } \\
\text { with CNN as feature extractor, } \\
\text { gives the best result RC, CCR, } \\
\text { DOR with a higher value. }\end{array}$ \\
\hline [29] & MIT - BIH arrhythmias & $\begin{array}{l}\text { Extracting Q-, R- T- and } \\
\text { U- waves }\end{array}$ & $\begin{array}{l}\text { Using BPNN to extract features } \\
\text { and classification. }\end{array}$ & $\begin{array}{l}\text { The proposed method gets } \\
98.70 \% \text { accuracy. }\end{array}$ \\
\hline$[30]$ & MIT - BIH arrhythmia & $\begin{array}{l}\text { STFT (Short-time Fourier } \\
\text { Transform) }\end{array}$ & 2D-CNN & $\begin{array}{l}\text { The average accuracy reaches } \\
99.00 \%\end{array}$ \\
\hline [31] & MIT - BIH arrhythmia & - & $\begin{array}{l}\text { Feature Extraction using CNN } \\
\text { and RNN for classification }\end{array}$ & $\begin{array}{l}\text { Proposed method obtain } 95.90 \% \\
\text { accuracy, } 95.90 \% \text { sensitivity and } \\
96.34 \% \text { specificity }\end{array}$ \\
\hline$[32]$ & MIT-BIH Arrhythmia & - & $\begin{array}{lll}\begin{array}{l}\text { Single-layer } \\
\text { classification }\end{array} & \text { LSTM } & \text { for } \\
\end{array}$ & $\begin{array}{l}\text { The proposed method obtains } \\
95 \% \text { accuracy }\end{array}$ \\
\hline$[33]$ & MIMIC-II Dataset & Histogram analysis & $\begin{array}{l}\text { Feature extraction using CNN } \\
\text { and classification using LSTM }\end{array}$ & $\begin{array}{l}\text { The proposed method obtains } \\
93 \% \text { accuracy }\end{array}$ \\
\hline
\end{tabular}
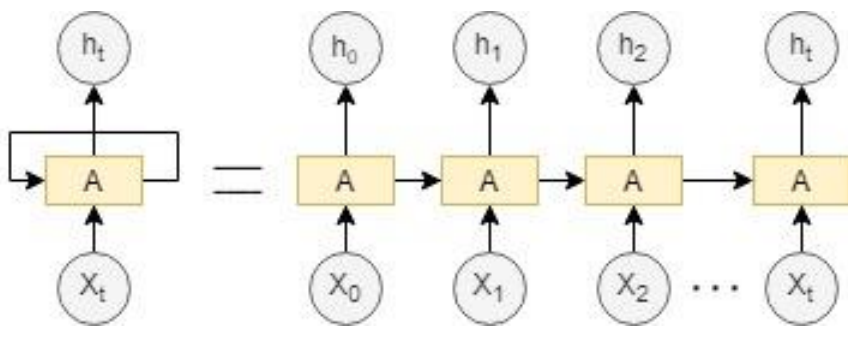

Fig. 6 Basic architecture of RNN.

Many researchers use CNN for ECG signal classification because CNN can give better accuracy. CNN can be used as a classifier and feature extractor as in [22], [23] or combined with another method as in [24], [25]. In [22], [23], they do not use any other feature extraction or pre-processing method. It can be done because CNN can extract features. The study [24] used SMOTE to synthesize the signal in the pre-processing stage then using the CNN classifier. In [25], there was a combined STFT method and CNN.

\section{Recurrent Neural Network}

RNNs or Recurrent Neural Networks is a class of neural networks that has directed connections between units of an individual layer, which will exhibit the unit's temporal behavior. It means that the state of the current instance will affect the state of the next instance [19]. Fig. 6 shows the basic architecture of RNN. RNN maintains a hidden vector $h$, which is updated at time step $t$ as follows.

$$
h_{t}=\tanh \left(W h_{t-1}+I x_{t}\right) .
$$

$W$ is the recurrent weight matrix, tanh is the hyperbolic tangent function, and $I$ is a projection matrix. The hidden state $h$ is used to make a prediction.

$$
y_{t}=\operatorname{softmax}\left(W h_{t-1}\right) .
$$

Function softmax provides a normalized probability distribution over the possible classes, $\sigma$ is the logistic sigmoid function, and $W$ is a weight matrix. By using $h$ as the input to another RNN, RNNs can be stacked, creating deeper architectures.

$$
h_{t}^{l}=\sigma\left(W h_{t-1}^{l}+I h_{t}^{l-1}\right)
$$

Long Short-Term Memory Networks, usually called LSTMs and improved through the general recurrent neural networks, are capable of learning long-term dependencies [19]. Basically, 
there are three kinds of gates in the LSTM. They are the input gate, the forget gate, and the output gate

\section{E. Classification Method Comparison}

Research on ECG classification methods has been widely carried out, starting from the pre-processing stage, feature extraction, and the classification method. The methods are very diverse; for example, in the feature extraction stage, the wavelet transform method in the pre-processing stage using SMOTE algorithm or Pan-Tomkin's algorithm can be used. For the classification method, SVM, K-NN, and Neural Network can be employed. Deep learning methods such as CNN and RNN are most commonly used because they have proven to provide high accuracy results. These methods can be seen in Table I.

\section{DISCUSSION AND CONCLUSION}

This paper discusses some of the classification methods that are most frequently used to classify ECG signals. In general, there are two stages of ECG classification, namely, the feature extraction stage and the classification stage. Before extracting features, the raw ECG signal data was processed first in the preprocessing stage since ECG signals were not necessarily free of noise. Noise will cause a decrease in accuracy during the classification process. After going through the pre-processing stage, feature extraction could be performed using various extraction methods, such as the Hadamard Transform feature Extraction in [27] or DWT in [29]. Deep learning methods such as CNN and RNN do not need to extract features manually because a deep neural network can extract its own features. The extracted features were then processed using the learning method to obtain accurate classification results. After getting the classification results, the validation of these results was performed; the most commonly used validation is the confusion matrix.

The confusion matrix is a technique in data mining to measure an algorithm's performance, and it is usually used after training and testing the algorithm. The estimated classification must have one of the four ranks, as shown in Table I.

The accuracy formula is:

$$
\text { Accuracy }=\frac{T P+T N}{T P+F P+F N+T N} \text {. }
$$

The precision value of the classification of true value is obtained based on (4).

$$
\text { Precision }=\frac{T P}{T P+F P} .
$$

The sensitivity of the model is obtained by (5).

$$
\text { Sensitivity }=\frac{T P}{T P+F N} \text {. }
$$

Based on Table II, the proposed method will obtain a confusion matrix value to determine its accuracy, sensitivity, and precision.

Based on the discussion and conclusion above, neural network methods such as CNN and RNN are the best and very popular to be used for ECG classification because they have shown to have high accuracy but with low complexity. It is because a deep learning method such as $\mathrm{CNN}$ can extract its
TABLE II

CONFUSION MATRIX TABLE

\begin{tabular}{|c|c|l|l|c|}
\hline \multicolumn{2}{|c|}{} & \multicolumn{2}{|l|}{ Predicted Values } & \multirow{2}{*}{ Total } \\
\cline { 3 - 4 } \multicolumn{2}{|c|}{$\begin{array}{c}\text { Yes } \\
\text { (positive) }\end{array}$} & $\begin{array}{c}\text { No } \\
\text { (negative) }\end{array}$ & \\
\hline $\begin{array}{c}\text { Actual } \\
\text { Values }\end{array}$ & Yes (positive) & TP & FN & TP + FN \\
\cline { 2 - 5 } & No (negative) & FP & TN & FP + TN \\
\hline & Total & TP + TF & FN + TN & \\
& & & \multicolumn{2}{c}{} \\
\end{tabular}

own features without the need of using other feature extraction methods. In the study [22], CNN was able to get an accuracy of 93.6\% without going through the pre-processing stage. Although the deep neural network has higher accuracy than the conventional method, for some multi-class classification above 5 classes, some class precision is quite low. For example, in [26], some class arrhythmia's precision was quite low at $86 \%$ even though the accuracy were above $90 \%$. It happens because some beat in arrhythmia database almost has the same characteristic waveform.

For further research, the machine learning method needs to be improved to get high accuracy and high precision to give an accurate diagnosis; this can be achieved with better precision to differentiate each beat without going through complicated preprocessing stages.

\section{REFERENCES}

[1] N. Joseph (2018) "Hello Sehat," [Online], www.hellosehat.com/pusatkesehatan/serangan-jantung/6-tips-kesehatan-jantung-dari-para-ahlijantung-ternama, access date: 24-Feb-2019.

[2] (2019) "IHME" [Online], http://www.healthdata.org, access date: 28Feb-2019.

[3] J.S. Walker, Wavelet and Their Scientific Applications, Boca Raton, USA: CRC Press, 1999.

[4] B.N. Hung, Y.S. Tsai, and T.H. Chu, "FFT Algorithm for PVC Detection Using IBM PC," Proc. $8^{\text {th }}$ Ann. Int. Conf. of the IEEE Eng. in Med. and Biol. Soc., 1986, pp. 292-295.

[5] B.N. Hung, H.F. Cheng, and Y.S. Tsai, "An Application of Fast Walsh Transform in ECG Diagnosis," Proc. $9^{\text {th }}$ Ann. Int. Conf. of the IEEE Eng. in Med. and Biol. Soc., 1987, pp. 497-498.

[6] J. Nadal and R.B. Panerai, "Classification of Cardiac Arrhythmias Using Principal Component Analysis of the ECG," Proc. of the Ann. Int. Conf.of the IEEE Eng. Med. and Biol. Soc., Vol. 13, 1991, pp. 580-581.

[7] W.H. Chang, K.P. Lin, and S.Y. Tseng, "ECG Analysis Based on Hilbert Transform Descriptor," Proc. 10 ${ }^{\text {th }}$ Ann. Int. Conf. of the IEEE Eng. in Med. and Biol. Soc., 1988, pp. 36-37.

[8] N.V. Thakor and Y.S. Zhu, "Applications of Adaptive Filtering to ECG Analysis: Noise Cancellation and Arrhythmia Detection," IEEE Trans. Biomed. Eng., Vol. 38, No. 8, pp. 785-794, 1991.

[9] D.A. Coast, R.M. Stem, G.G. Cano, and S.A. Briller, "An Approach to Cardiac Arrhythmia Analysis Using Hidden Markov Models," IEEE Trans. Biomed, Eng., Vol. 37, No. 9, pp. 826-836, 1990

[10] S. Osowsaki and T. Linh, "ECG Beat Recognition Using Fuzzy Hybrid Neural Network," IEEE Trans. Biomed. Eng., Vol. 48, No. 11, pp. 12651271, 2001.

[11] L. He, W. Hou, X. Zhen, and C. Peng, "Recognition of ECG Patterns Using Artificial Neural Network", Proc. of the Sixth Int. Conf. on Intel. Syst. Design and Appl. (ISDA'06), 2006, pp. 1-5.

[12] M.F.M. Elias and H. Arof, "Classification of Electrocardiogram Signal Using Multiresolution Wavelet Transform and Neural Network," Proc. $3^{\text {rd }}$ Kuala Lumpur Int. Conf. on Biomed. Eng., 2006, pp. 360-364.

[13] N. Maglaveras, T. Stamkopoulos, K. Diamantaras, C. Pappas, and M. Strintzis, "ECG Pattern Recognition and Classification Using Non-Linear Transformations and Neural Networks: A Review," Int. J. Med. Inform, Vol. 52, No. 1-3, pp. 191-208, 1998. 
[14] T.-H. Chen, Y. Zheng, L.-Q. Han, P.-Y. Guo, and X.-Y. He, "The Sorting Method of ECG Signals Based on Neural Network," Proc. Int. Conf. on Bioinform. and Biomed. Eng., 2008, pp. 543-546.

[15] H.M. Rai and A. Trivedi, "ECG Signal Classification Using Wavelet Transform and Back Propagation Neural Network," Proc. $5^{\text {th }}$ Int. Conf. on Comput. and Dev. for Commun., 2012, pp. 1-4.

[16] V. Seena and J. Yomas, "A Review on Feature Extraction and Denoising of ECG Signal Using Wavelet Transform," Proc. $2^{\text {nd }}$ Int. Conf. on Devices, Circ. and Syst., 2014, pp.1-6.

[17] M.H.F.M. Jalil, M.F. Saaid, A. Ahmad, and M.S.A.M. Ali, "Arrhythmia Modeling via ECG Characteristic Frequencies and Artificial Neural Network," Proc. 2014 IEEE Conf. on Syst., Process, and Control (ICSPC 2014), 2014, pp. 121-126.

[18] K.J. Chen and P. Chien, "A Fast ECG Diagnosis Using Frequency-based Compressive Neural Network," Proc. 2017 IEEE 6th Glob. Conf. on Consum. Electron. (GCCE), 2017, pp. 3-4.

[19] F. Karim, S. Majumdar, H. Darabi, and S. Chen, "LSTM Fully Convolutional Networks for Time Series Classification," IEEE Access, Vol. 6, pp. 1662-1669, 2018.

[20] J.J. Siang, Jaringan Syaraf Tiruan dan Pemrogramannya Menggunakan Matlab, Yogyakarta, Indonesia: ANDI OFFSET, 2005.

[21] A. Hidaka and T. Kurita, "Consecutive Dimensionality Reduction by Canonical Correlation Analysis for Visualization of Convolutional Neural Networks," Proc. of the ISCIE Int. Symp. on Stoch. Syst. Theory and Its Appl., 2017, pp. 160-167.

[22] A. Rajkumar, M. Ganesan, and R. Lavanya, "Arrhythmia Classification on ECG Using Deep Learning," 2019 5th Int. Conf. on Adv. Comp. \& Commun. Syst. (ICACCS), 2019, pp. 365-369.

[23] S. Savalia and V. Emamian. "Cardiac Arrhythmia Classification by Multi-Layer Perceptron and Convolution Neural Networks," Bioengineering, Vol. 5, No. 2, pp. 1-12, 2018.

[24] F. Liu, X. Zhou, J. Cao, Z. Wang, H. Wang, and Y. Zhang, "A LSTM and CNN Based Assemble Neural Network Framework for Arrhythmias
Classification," Proc. 2019 IEEE Int. Conf. on Acoust., Speech and Sign. Proces. (ICASSP), 2019, pp. 1303-1307.

[25] S. Kiranyaz, T. Ince, and M. Gabbouj, "Real-time Patient-specific ECG Classification by 1-D Convolutional Neural Networks," IEEE Trans. Biomed. Eng., Vol. 63, No. 3, pp. 664-675, 2016.

[26] S. Saadatnejad, M. Oveisi, and M. Hashemi, "LSTM-Based ECG Classification for Continuous Monitoring on Personal Wearable Devices," IEEE J. of Biomed. and Health Inform., Vol. 24, No. 2, pp. 515 523, Feb. 2020.

[27] A. Amirshahi and M. Hashemi, "ECG Classification Algorithm Based on STDP and R-STDP Neural Networks for Real-time Monitoring on Ultra Low-Power Personal Wearable Devices," IEEE Trans. Biomedi. Circ. and Syst. (TBioCAS), Vol. 13, No. 6, pp. 1483-1493, Dec. 2019.

[28] B. Pourbabaee, M.J. Roshtkhari, and K. Khorasani, "Deep Convolutional Neural Networks and Learning ECG Features for Screening Paroxysmal Atrial Fibrillation Patients," IEEE Tran. on Syst., Man, and Cybernetics: Systems, Vol. 48, No. 12, pp. 2095-2104, Dec. 2018.

[29] E. Al-masri, "Detecting ECG Heartbeat Abnormalities Using Artificial Neural Networks," Proc. IEEE Int. Conf. on Big Data, 2018, pp. 52795281.

[30] J. Huang, B. Chen, B. Yao, and W. He, "ECG Arrhythmia Classification Using STFT-Based Spectrogram and Convolutional Neural Network," IEEE Access, Vol. 7, pp. 92871-92880, 2019.

[31] X. Xu, S. Jeong, and J. Li, "Interpretation of Electrocardiogram (ECG) Rhythm by Combined CNN and BiLSTM," IEEE Access, Vol. 8, pp. 125380-125388, 2020

[32] A. Rana and K.K. Kim, "ECG Heartbeat Classification Using a Single Layer LSTM Model,” Proc. 2019 Int. SoC Design Conf. (ISOCC), 2019, pp. 267-268.

[33] R. Banerjee, A. Ghose, and K.M. Mandana, "A Hybrid CNN-LSTM Architecture for Detection of Coronary Artery Disease from ECG," Proc. 2020 Int. Joint Conf. on Neural Networks (IJCNN), 2020, pp. 1-8. 